\title{
Detecting tropical wildlife declines through camera- trap monitoring: an evaluation of the Tropical Ecology Assessment and Monitoring protocol
}

\author{
Lydia Beaudrot, Jorge Ahumada, Timothy G. O’Brien and Patrick A. Jansen
}

\begin{abstract}
Identifying optimal sampling designs for detecting population-level declines is critical for optimizing expenditures by research and monitoring programmes. The Tropical Ecology Assessment and Monitoring (TEAM) network is the most extensive tropical camera-trap monitoring programme, but the effectiveness of its sampling protocol has not been rigorously assessed. Here, we assess the power and sensitivity of the programme's camera-trap monitoring protocol for detecting occupancy changes in unmarked populations using the freely available application PowerSensor!. We found that the protocol is well suited to detect moderate $(\geq 5 \%)$ population changes within 3-4 years for relatively common species that have medium to high detection probabilities (i.e. $\mathrm{p}>0.2$ ). The TEAM protocol cannot, however, detect typical changes in rare and evasive species, a category into which many tropical species and many species of conservation concern fall. Additional research is needed to build occupancy models for detecting change in rare and elusive species when individuals are unmarked.
\end{abstract}

Keywords Camera trap, conservation, monitoring, power analysis, sampling design, Tropical Ecology Assessment and Monitoring, wildlife management

Supplementary material for this article is available at https://doi.org/10.1017/So030605318000546

amera-trap surveys have become a popular technique $\checkmark$ for assessing change in wildlife populations (O'Brien, 2016). The Tropical Ecology Assessment and Monitoring (TEAM) network is the most extensive tropical camera-trap

Lydia BEAUdRot (Corresponding author) Department of Ecology and Evolutionary Biology, Michigan Society of Fellows, University of Michigan, 830 University Avenue, Ann Arbor, Michigan 48108, USA

E-mail beaudrot@umich.edu

JoRGe AHUMADA* Moore Center for Science, Conservation International, Arlington, Virginia, USA

Тімотну G. O’Brien Global Conservation Program, Wildlife Conservation Society, Bronx, New York, USA

Patrick A. Jansen† Center for Tropical Forest Science, Smithsonian Tropical Research Institute, Balboa, Ancon, Panamá

${ }^{*}$ Also at: Center for Biodiversity Outcomes, Arizona State University, Tempe, Arizona, USA

$\dagger$ Also at: Department of Environmental Sciences, Wageningen University, Wageningen, The Netherlands

Received 7 August 2017. Revision requested 23 November 2017.

Accepted 12 April 2018. First published online 10 September 2018. programme, monitoring terrestrial wildlife in 17 tropical forests. The programme's mission is to provide an early warning system for the status of biodiversity by monitoring $>500$ populations of ground-dwelling mammals and birds (Beaudrot et al., 2016). The camera-trap data have been used to evaluate wildlife trends within and across protected areas and to assess the effectiveness of protected areas in maintaining wildlife populations (Ahumada et al., 2011, 2013; Beaudrot et al., 2016). However, the power of TEAM's protocol to detect occupancy changes has not been assessed. Like many camera-trap monitoring programmes, TEAM has hitherto relied on rules of thumb and common practices for survey design, in particular for determining the number of points to survey and the duration of sampling at each point. Specifically, TEAM has deployed camera traps across a grid of 60-90 points at a density of $1-2$ per $\mathrm{km}^{2}$ in each forest. Field assistants activate the cameras annually for 1 month (30 sampling days).

Here, we assess the power and sensitivity of the programme's sampling design and its camera-trap monitoring protocol (TEAM Network, 2011; Jansen et al., 2014). We use PowerSensor! (TEAM Network, 2018) to calculate the sensitivity of wildlife occupancy trends based on the number of sampling points and the sampling duration for populations with varying levels of initial occupancy and detectability. Specifically, we assess the sensitivity of the TEAM protocol to annual linear occupancy declines ranging from severe (15\%) to small (1\%; Fig. 1).

We estimated the initial occupancy of each of the 511 populations monitored by TEAM using the modelling output of the first global assessment of wildlife trends, which utilized camera-trap data collected during 2007-2014 (Beaudrot et al., 2016). Similarly, we calculated detection probabilities for each population using the same dataset. We defined the number of years required to detect change using a conservative cutoff of an $80 \%$, rather than the typical $95 \%$, confidence interval for classifying occupancy trends, because a wider confidence interval can provide an earlier warning signal of occupancy declines that can prompt conservation action (Myers, 1993). We then used PowerSensor! (2018) to determine the number of years necessary to detect change for each population based on its initial occupancy and detection probabilities and an effort of 60 points with 30 days of sampling.

PowerSensor! displays information that we generated through a simulation and statistical analysis, which we 


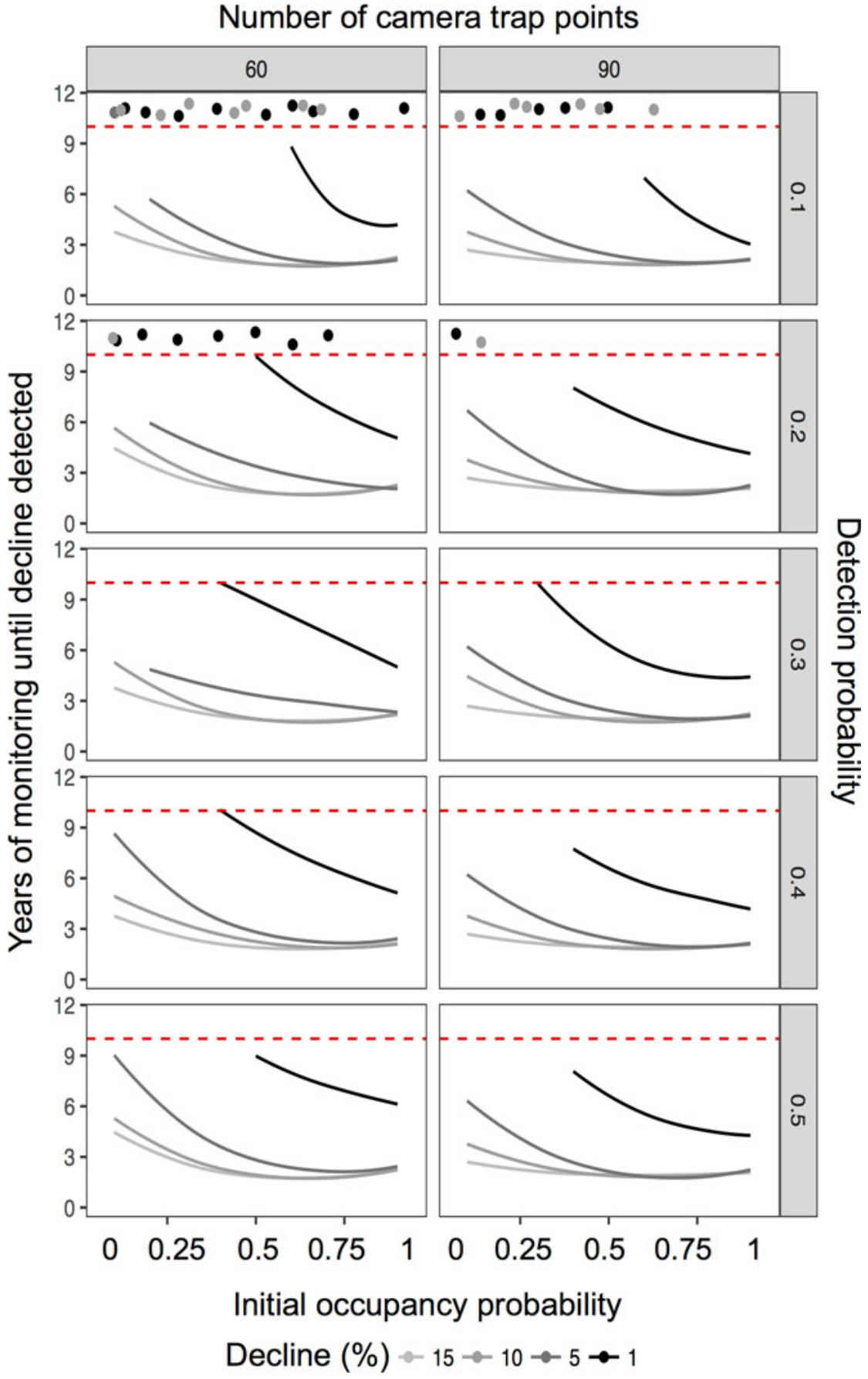

FIg. 1 Sensitivity of the TEAM camera-trap protocol, expressed as the number of years of sampling required to detect annual occupancy declines of 15, 10, 5 and $1 \%$, given an effort of 60 or 90 camera traps sampling for 30 days annually, for species with initial occupancy probabilities of 0.1 to 0.9 and detection probabilities of 0.1 to 0.5 . Not all declines could be detected within 10 years, particularly small declines (i.e. $1 \%$ ), which resulted in shorter lines graphed in the figure. Declines that were not detectable within 10 years are shown with points above the dashed line, which demarcates the 1oth year. briefly describe here. Firstly, we simulated data for declining species using the dynamic occupancy model formulated by MacKenzie et al. (2003).We generated time series data, varying the following parameters: number of sites, number of times a site is sampled within a survey, occupancy probability in the first survey, detection probability and persistence probability. The colonization probability and the number of surveys were fixed. We intentionally set the colonization probability to zero to simulate population declines as the worst-case scenario. We set the number of surveys to 10 because many biodiversity policy framework plans (e.g.
Convention on Biological Diversity) measure progress in 10-year intervals (Butchart et al., 2010). We did not model covariates on initial occupancy, extinction, colonization or detection probability. The parameters did not vary between sites, samples within a survey, or surveys. Once the data were generated, we fitted the same dynamic occupancy model using the colext function of the unmarked package (Fiske \& Chandler, 2011) in $R$ 3.5.o (R Development Core Team, 2016).

Secondly, for each simulation and parameter combination, we fitted the model without covariates and projected 
the model trajectory under a finite number of sites using the smoothed model projection of unmarked:

$$
\hat{Z}_{k, m, t}=\hat{Z}_{k, m, t-1} \Psi_{m},
$$

where $\hat{Z}_{k, m, t}$ is the estimated occupancy from a simulated data set $k$ with parameter combination $m$ at survey $t$, and $\psi$ is the probability of a site being occupied.

Thirdly, to assess whether the fitted models were able to detect change under a given parameter combination $m$ and after two surveys, we calculated the quantity $\hat{Z}_{k, m, 1}-\hat{Z}_{k, m, t}$ for each of the $250 \mathrm{~m}$ fitted models and surveys $t(t>1)$. For each survey $t$, we obtained a distribution of 250 difference values and tested whether the 80,90 or $95 \%$ confidence intervals of the distribution included the value zero. If so, the model was unable to detect the simulated changes in occupancy for that particular survey given the confidence level. All simulations and analyses were conducted in $R$, and the code is available on Github (Ahumada, 2017).

In general, higher rates of decline could be detected more often and within fewer years than lower rates of decline; declines were less detectable when detection probabilities and/or initial occupancy probabilities were low. We found that just 2 years of sampling with 60 camera-trap points was sufficient to detect severe (15\%) annual occupancy declines for populations with initial occupancy probabilities $\geq 0$.3. For populations with lower initial occupancies (i.e. $\psi=0.1)$, severe $(15 \%)$ declines were generally detectable with 3 years of sampling and 90 camera-trap points.

To detect $10 \%$ annual occupancy declines, 2 years of sampling with 60 camera traps was sufficient for populations with initial occupancy probabilities $\geq 0.5$. For $\psi=0.3$, 3 years of sampling with 60 camera-trap points was sufficient, and for $\psi=0.1,10 \%$ declines were detectable with 5 years of sampling and 90 camera-trap points.

For $5 \%$ annual occupancy declines the number of years of sampling necessary to detect declines was more contingent on the initial occupancy probability than for 15 and 10\% declines. Similarly, the likelihood that declines were detectable was more contingent on the detection probability. Generally, 5\% annual declines were detectable with 4 years of sampling using 60 camera-trap points or with 3 years of sampling using 90 camera-trap points for populations with initial occupancy probabilities $\geq 0.3$ and detection probabilities $\geq 0.2$. Finally, a small (1\%) decline was consistently detected within 10 years for detection probability $=0.5$. For initial occupancy $\psi=0.5$ a small decline was detected in 10 years, for initial occupancy $\psi=0.7$ in 6 years, and for initial occupancy $\psi=0.9$ in 5 years.

The majority of the 511 populations that the programme monitors did not meet the initial occupancy probability threshold of 0.1 and detection probability threshold of 0.04 necessary to detect change within 10 years using PowerSensor! (Fig. 2). Specifically, the initial occupancy probabilities for 274 populations $(53.6 \%)$ were $<0.1$ and detection probabilities
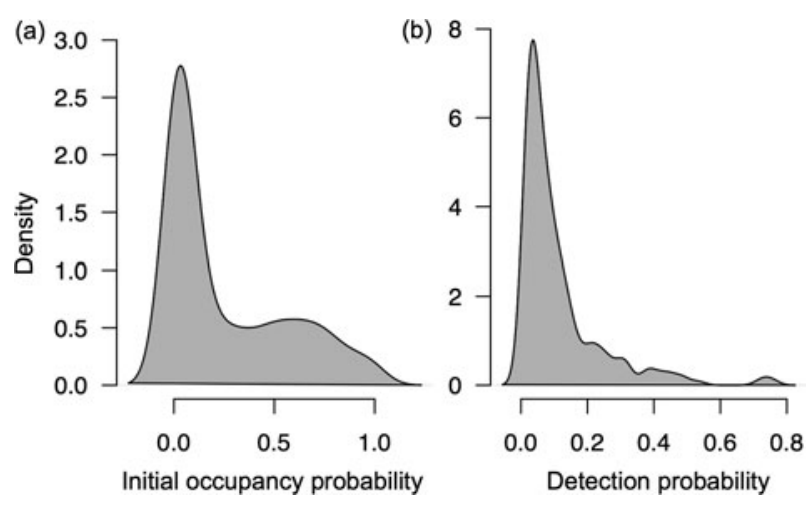

FIG. 2 Density plots of the (a) initial occupancy probabilities ( $\mathrm{N}=511$ populations) and (b) estimated detection probabilities $(\mathrm{N}=233$ populations) for the terrestrial mammal and bird populations that TEAM monitors. The remaining 278 populations had $<5$ camera-trap detections per year and therefore had insufficient observations to estimate detection probabilities.

for 359 populations (70.3\%) were $<0.04$. We were able to assess the percentage of detectable change for $150(29.3 \%)$ of the 511 populations (Supplementary Table 1). Occupancy changes of 15 and $10 \%$ were detectable for all 150 populations within 5 years and 7 years, respectively. Occupancy changes of $5 \%$ were detectable for 130 populations within 7 years and changes of $1 \%$ were detectable for 80 populations within 10 years. Of these 150 populations, $85.33 \%$ were mammals and $14.67 \%$ were birds. The majority were herbivores $(45.3 \%)$ or omnivores $(44.0 \%)$, and few were carnivores $(8.67 \%)$ or insectivores $(2.0 \%)$. According to the IUCN (2014) Red List, the majority of the 150 populations are categorized as Least Concern (76.0\%), followed by Vulnerable (8.0\%), Near Threatened $(5.33 \%)$, Endangered (5.33\%), Data Deficient (4.67\%) and Critically Endangered (0.67\%; Supplementary Table 1).

Our examination reveals the relative power and sensitivity of the TEAM protocol to detect annual changes in occupancy given currently available single-species occupancy models for unmarked individuals. The protocol is well suited to detect moderate $(\geq 5 \%)$ changes in occupancy within 4 years for common tropical species (i.e. initial occupancy $>0.3)$ that have medium to high detection probabilities (i.e. $\mathrm{p}>$ o.2). The TEAM protocol cannot, however, detect the typical changes in occupancy of rare and evasive species, a category in which most tropical species and many species of conservation concern fall. This is a challenge faced not only by camera trapping but also by many other wildlife monitoring techniques (Ellison \& Agrawal, 2005; MacKenzie et al., 2005).

Multi-species models collectively model all species within a community while still allowing each species to respond individually to sampling variables (Dorazio et al., 2006; Zipkin et al., 2009). Such models can provide more precise estimates of occupancy for species that have been observed 
less often, by formally sharing data across species. To date, multi-species models have been limited in their utility to communities with few rare species or have required the exclusion of the rarest species (Ruiz-Gutierrez et al., 2010). Nevertheless multi-species models may offer a promising method for improving occupancy estimates compared to what is possible with single species models. Additional research is needed to build models for detecting change in rare and elusive species when individuals are unmarked.

Acknowledgements We thank Eric Fegraus and James McCarthy for technical support, Alex Zvoleff and Elise Zipkin for discussions, and the anonymous reviewers for their critiques.

Author contributions Analyses, writing and creation of figures: LB, with contributions from all authors; development of PowerSensor!: JA, with contributions from all authors.

\section{Conflicts of interest None.}

Ethical standards This research complied with the Oryx Code of Conduct for authors.

\section{References}

Ahumada, J. (2017) Https:/github.com/jaahumadap/PAOccPaper/ tree/master/ShinyApps/powerPaper [accessed 14 June 2018].

Ahumada, J.A., Hurtado, J. \& Lizcano, D. (2013) Monitoring the status and trends of tropical forest terrestrial vertebrate communities from camera trap data: a tool for conservation. PLoS ONE, 8, e73707.

Ahumada, J.A., Silva, C.E.F., Gajapersad, K., Hallam, C., Hurtado, J., Martin, E. et al. (2011) Community structure and diversity of tropical forest mammals: data from a global camera trap network. Philosophical Transactions of the Royal Society B: Biological Sciences, 366, 2703-2711.

Beaudrot, L., Ahumada, J.A., O'Brien, T., Alvarez-Loayza, P., Boekee, K., Campos-Arceiz, A. et al. (2016) Standardized assessment of biodiversity trends in tropical forest protected areas: the end is not in sight. PLoS Biology, 14, e1002357.

Butchart, S.H.M., Walpole, M., Collen, B., van Strien, A., Scharlemann, J.P.W., Almond, R.E.A. et al. (2010)
Global biodiversity: indicators of recent declines. Science, 328, 11641168 .

Dorazio, R.M., Royle, J.A., Soderstrom, B. \& Glimskar, A. (2006) Estimating species richness and accumulation by modeling species occurrence and detectability. Ecology, 87, 842-854.

Ellison, A.M. \& Agrawal, A.A. (2005) The statistics of rarity. Ecology, 86, 1079-1080.

Fiske, I.J. \& ChandleR, R.B. (2011) unmarked: An $R$ package for fitting hierarchical models of wildlife occurrence and abundance. Journal of Statistical Software, 43, 1-23.

IUCN (2014) The IUCN Red List of Threatened Species v. 2014.1. Http:// www.iucnredlist.org [accessed 7 April 2014].

Jansen, P. A., Ahumada, J., Fegraus, E. \& O’Brien, T. (2014) TEAM: a standardised camera trap surey to monitor terrestrial vertebrate communities in tropical forests. In Camera Trapping: Wildlife Management and Research (eds P. Meek \& P. Fleming), pp. 263-27o. CSIRO Publishing, Collingwood, Australia. MacKenzie, D.I., Nichols, J.D., Hines, J.E., Knutson, M.G. \& FrankLIN, A.B. (2003) Estimating site occupancy, colonization, and local extinction when a species is detected imperfectly. Ecology, 84, $2200-2207$.

MacKenzie, D.I., Nichols, J.D., Sutton, N., Kawanishi, K. \& BAILEY, L.L. (2005) Improving inferences in popoulation studies of rare species that are detected imperfectly. Ecology, 86, 1101-1113.

Myers, N. (1993) Biodiversity and the precautionary principle. Ambio, 22, 74-79.

O'BRIEN, T. (2016) Camera traps for conservation: monitoring protected area investments. In Protected Areas: Are They Safeguarding Biodiversity? (eds L. N. Joppa, J. G. Robinson \& I. Baillie), pp. 228-241. Wiley-Blackwell, London, UK.

R Development Core Team (2016) R: A Language and Environment for Statistical Computing. R Foundation for Statistical Computing, Vienna, Austria.

Ruiz-Gutierrez, V., Zipkin, E.F. \& Dhondt, A.A. (2010) Occupancy dynamics in a tropical bird community: unexpectedly high forest use by non-forest bird species. Journal of Applied Ecology, 47, 621-630.

TEAM Network (2011) Tropical Ecology Assessment \& Monitoring Network. Http://www.teamnetwork.org [accessed 1 June 2016].

TEAM Network (2018) PowerSensor! Http://analytics.teamnetwork. org/jahumada/powerPaper [accessed 1 August 2017].

Zipkin, E.F., DeWan, A. \& Royle, J.A. (2009) Impacts of forest fragmentation on species richness: hierarchical approach to community modelling. Journal of Applied Ecology, 46, 815-822. 